\title{
THE UNITARY EQUIVALENCE OF COMPACT OPERATORS
}

\author{
by WAI-FONG CHUAN
}

(Received 18 April, 1984)

1. Introduction. The problem of determining whether or not two operators are unitarily equivalent has been around for many years and considerable work has been done in attempting to solve this problem (see for example [1], [3], [5], [6], [7], [10], [11], [12], $[\mathbf{1 5}],[\mathbf{1 6}],[\mathbf{1 7}],[\mathbf{1 8}],[\mathbf{1 9}],[\mathbf{2 0}],[\mathbf{2 1}]$ and $[\mathbf{2 2}])$. In many cases, a complete set of unitary invariants is furnished for a certain class of operators. Here we just mention two of such results which are related to what we are going to discuss. The first one was due to Arveson, who showed that two irreducible compact operators are unitarily equivalent if and only if they have the same $n$th algebraic matricial ranges, for each $n \geqq 1$ ([1] and Theorem 2.4.3 of [3]). The second one was due to Parrott, who showed that two compact operators with zero reducing null spaces are unitarily equivalent if and only if they have the same $n$th spatial matricial ranges, for each $n \geqq 1([5$, p. 146]). In this paper, we investigate the closures of the spatial matricial ranges of compact operators and obtain a complete set of unitary invariants for compact operators, from which Parrott's result follows easily.

2. Main theorems. Two operators $S$ and $T$ acting on a Hilbert space $H$ are said to be unitarily equivalent if there exists a unitary operator $U$ on $H$ such that $S=U^{*} T U$; they are approximately equivalent if there is a sequence $\left\{U_{n}\right\}$ of unitary operators such that $\left\{U_{n}^{*} T U_{n}\right\}$ converges to $S$ in norm (cf. [13]).

The purpose of this paper is to prove the following two theorems.

THEOREM 1. Two compact operators on an infinite-dimensional separable Hilbert space are approximately equivalent if and only if, for each $n \geqq 1$, their nth spatial matricial ranges have the same closure.

THEOREM 2. Two compact operators on an infinite-dimensional separable Hilbert space are unitarily equivalent if and only if their reducing null-spaces have the same dimension and, for each $n \geqq 1$, their nth spatial matricial ranges have the same closure.

3. Notations. Let $H$ and $K$ be two Hilbert spaces. We denote by $\mathscr{L}(H, K)$ the Banach space of all (bounded) linear transformations from $H$ into $K$, and by $\mathscr{L}(H)$ the $C^{*}$-algebra of all (bounded) operators on $H$. For $r>0$, let $\mathscr{L}_{r}(H, K)$ be the set of all $S$ in $\mathscr{L}(H, K)$ whose norm is less than or equal to $r$, and let $\mathscr{L}_{r}(H)$ be defined similarly.

A net $\left\{\zeta_{\alpha}\right\}$ of vectors in $H$ is said to converge to the vector $\zeta$ weakly if $\lim _{\alpha}\left(\zeta_{\alpha}, \eta\right)=$ $(\zeta, \eta)$, for all $\eta$ in $H$, where $(\cdot, \cdot)$ denotes the inner product. Let $\left\{F_{\alpha}\right\}$ be a net in $\mathscr{L}(H, K)$. We say that $\left\{F_{\alpha}\right\}$ converges to an operator $F$ strongly (respectively, weakly) if $\left\{F_{\alpha} \zeta\right\}$ converges to $F \zeta$ strongly (respectively, weakly) in $K$, for all $\zeta$ in $H$.

If $\mathscr{A}$ is a unital $\mathrm{C}^{*}$-algebra, we denote by $\mathrm{CP}(\mathscr{A}, H)$ the set of all unital completely

Glasgow Math. J. 26 (1985) 145-149. 
positive linear maps of $\mathscr{A}$ into $\mathscr{L}(H)$. Given a positive integer $n$, let $H_{n}$ denote the $n$-dimensional Hilbert space of all $n$-tuples of complex numbers. We identify the $n$ by $n$ matrices with the operators on $H_{n}$. For $T$ in $\mathscr{L}(H)$, let

$$
\begin{aligned}
& W_{n}(T)=\left\{V^{*} T V: V \text { is an isometry from } H_{n} \text { into } H\right\}, \\
& W_{n}(T)=\left\{\phi(T): \phi \in \operatorname{CP}\left(C^{*}(T), H_{n}\right)\right\},
\end{aligned}
$$

where $C^{*}(T)$ denotes the unital $C^{*}$-subalgebra of $\mathscr{L}(H)$ generated by $T$ and the identity operator.

The sets $W_{n}(T)$ and $W_{n}(T)$ are called the nth spatial matricial ranges and the nth algebraic matricial ranges of $T$ respectively $\left([1],[3]\right.$ and [5]). Note that $W_{1}(T)$ equals the numerical range of $T$ and $W_{1}(T)$ equals the closure of $W_{1}(T)$. So we may regard these matricial ranges as generalizations of the numerical range and its closure.

4. Proofs. For the rest of this paper, $H$ will denote an infinite-dimensional separable Hilbert space. We first consider the closures of the spatial matricial ranges of a compact operator on $H$. The proof of the following lemma employs one of the deep results on $\mathrm{C}^{*}$-algebras due to Voiculescu [23].

Lemma 1. Let $T$ be a compact operator on $H$, let $K$ be a finite-dimensional or infinite-dimensional separable Hilbert space and let $S \in \mathscr{L}(K)$. Then the following conditions on $S$ are equivalent. into $\mathrm{H}$.

(i) $S$ is a norm limit of a sequence $\left\{V_{n}^{*} T V_{n}\right\}$, where each $V_{n}$ is an isometry from $K$

(ii) $S$ is a weak limit of a net $\left\{V_{\alpha}^{*} T V_{\alpha}\right\}$, where each $V_{\alpha}$ is an isometry from $K$ into $H$.

(iii) $S=F^{*} T F$, where $F \in \mathscr{L}_{1}(K, H)$.

Proof. The implication (i) $\Rightarrow$ (ii) is trivial.

(ii) $\Rightarrow$ (iii). Suppose $S$ is the weak limit of a net $\left\{V_{\alpha}^{*} T V_{\alpha}\right\}$, where each $V_{\alpha}$ is an isometry from $K$ into $H$. By the weak compactness of $\mathscr{L}_{1}(K, H)$, the net $\left\{V_{\alpha}\right\}$ has a subnet $\left\{V_{\alpha_{\beta}}\right\}$ which converges weakly to some $F$ in $\mathscr{L}_{1}(K, H)$. The compactness of $T$ then implies that $\left\{V_{\alpha_{\beta}}^{*} T V_{\alpha_{\beta}}\right\}$ converges to $F^{*} T F$ weakly. Consequently $S=F^{*} T F$.

(iii) $\Rightarrow$ (i). Suppose that $S=F^{*} T F$, where $F$ is in $\mathscr{L}_{1}(K, H)$. Let $Q$ be the positive square root of $I_{K}-F^{*} F$. Define $\pi$ to be the ${ }^{*}$-representation of $C^{*}(T)$ in $K$ such that $\pi(T)$ is the zero operator on $K$ and $\pi\left(I_{H}\right)=I_{K}$. Put

$$
\theta(A)=F^{*} A F+Q \pi(A) Q
$$

for $A$ in $C^{*}(T)$. Then $\theta$ is in $\operatorname{CP}\left(C^{*}(T), K\right)$. Indeed, $\theta(A)=W^{*} \sigma(A) W$, where $W$ is the isometry from $K$ into $H \oplus K$ given by

for $\zeta$ in $K$, and

$$
W \zeta=F \zeta \oplus Q \zeta
$$

$$
\sigma=i d \oplus \pi
$$

where id is the identity representation of $C^{*}(T)$ on $H$. Note that $\sigma$ is approximately equivalent to id because $\pi$ annihilates the compact operators in $C^{*}(T)([\mathbf{4}],[\mathbf{2 3}])$. In other 
words there exist unitary operators $U_{n}(n \geqq 1)$ from $H \oplus K$ into $H$ such that $\left\{U_{n}^{*} T U_{n}\right\}$ converges to $\sigma(T)$ in norm. Thus $S=F^{*} T F=\theta(T)=W^{*} \sigma(T) W$ is the norm limit of $W^{*} U_{n}^{*} T U_{n} W$, so that (i) holds. This completes the proof.

Let $W(T)$ (respectively, $U(T)$ ) denote the set of all operators on $H$ of the form $V^{*} T V$, where $V$ is an isometry (respectively, a unitary operator) on $H$, and let (respectively, ${ }^{-w}$ ) denote the closure in norm (respectively, in the weak operator topology).

Corollary. Let $T$ be a compact operator on $H$. Then

and

$$
W_{n}(T)^{-}=\left\{F^{*} T F: F \in \mathscr{L}_{1}\left(H_{n}, H\right)\right\}
$$

$$
W(T)^{-}=W(T)^{-w}=U(T)^{-w}=\left\{G^{*} T G: G \in \mathscr{L}_{1}(H)\right\} .
$$

Proof. In view of Lemma 1, it remains to show that $W(T)^{-w}=U(T)^{-w}$. To this end, it suffices to prove that $W(T) \subset U(T)^{-w}$. Let $V$ be a given isometry on $H$ and let $\left\{e_{n}\right\}$ be an orthonormal basis for $H$. For each $n$, let $U_{n}$ be a unitary operator on $H$ with $U_{n} e_{k}=V e_{k}$, where $1 \leqq k \leqq n$. Then the sequence $\left\{U_{n}\right\}$ converges to $V$ strongly so that $\left\{U_{n}^{*} T U_{n}\right\}$ converges to $V^{*} T V$ weakly. This completes the proof.

Arveson [3, Theorem 2.4.2] showed that $\mathcal{W}_{n}(S) \subset \mathcal{W}_{n}(T)$ for all $n \geqq 1$ if and only if there exists $\phi$ in $\operatorname{CP}\left(C^{*}(T), H\right)$ such that $\phi(T)=S$. In Lemma 3 we shall prove a similar result for compact operators $S$ and $T$ on $H$; namely, $W_{n}(S)^{-} \subset W_{n}(T)^{-}$for all $n \geqq 1$ if and only if $S=F^{*} T F$, where $F$ is in $\mathscr{L}_{1}(H)$ (see also [5, p. 144]). We also note from the proof of Lemma 1 that $S$ is in fact the image of $T$ under a unital completely positive linear map which is of a particular form.

In the following two lemmas we assume that $S$ and $T$ are operators on $H$ and that $T$ is compact.

LEMMA 2. If $n$ is a positive integer and if $W_{n}(S)^{-} \subset W_{n}(T)^{-}$, then for each projection $P$ of $H$ of rank $n$, there exists $F$ in $\mathscr{L}_{1}(H)$ such that $P S P=F^{*} T F$.

Proof. Note that $P=W W^{*}$ for some isometry $W$ in $\mathscr{L}\left(H_{n}, H\right)$. Since $W^{*} S W$ belongs to $W_{n}(T)^{-}$, it follows from the corollary to Lemma 1 that there exists $V$ in $\mathscr{L}_{1}\left(H_{n}, H\right)$ such that $W^{*} S W=V^{*} T V$. Now let $F=V W^{*}$. Then $F$ belongs to $\mathscr{L}_{1}(H)$ and $P S P=F^{*} T F$.

Lemma 3. If $W_{n}(S)^{-} \subset W_{n}(T)^{-}$for all $n \geqq 1$, then $S$ is compact and $S \in W(T)^{-}$.

Proof. By Lemma 1, it suffices to show that $S=F^{*} T F$ for some $F$ in $\mathscr{L}_{1}(H)$. To this end, let $\left\{P_{n}\right\}$ be a sequence of projections of finite rank which converges to $I$ strongly. By Lemma 2, for each $n \geqq 1$, there exists $F_{n}$ in $\mathscr{L}_{1}(H)$ such that $P_{n} S P_{n}=F_{n}^{*} T F_{n}$. The sequence $\left\{F_{n}\right\}$ has a subnet $\left\{F_{n_{0}}\right\}$ which converges to some $F$ in $\mathscr{L}_{1}(H)$. Now since $T$ is compact, it follows that $F_{n_{\alpha}}^{*} T F_{n_{n}}$ converges weakly to $F^{*} T F$. On the other hand, the sequence $\left\{P_{n} S P_{n}\right\}$ converges to $S$ weakly. Hence $S=F^{*} T F$ and the result follows.

Corollary 1. Let $S$ and $T$ be two compact operators on $H$. Then $W_{n}(S)^{-}=W_{n}(T)^{-}$for all $n \geqq 1$ if and only if $W(S)^{-}=W(T)^{-}$. 
Proof. It is clear that the condition $W(S)^{-}=W(T)^{-}$implies that $W_{n}(S)^{-}=W_{n}(T)^{-}$for all $n \geqq 1$. The converse is an immediate consequence of Lemma 3 .

Combining Theorem 3 of [14] and the corollaries to Lemma 1 and 3 we arrive at the following result, from which Theorem 1 follows immediately.

Corollary 2. Let $S$ and $T$ be two compact operators on $H$. Then the following conditions are equivalent.

(i) $S$ is approximately equivalent to $T$.

(ii) $U(S)^{-}=U(T)^{-}$.

(ii') $U(S)^{-w}=U(T)^{-w}$.

(iii) $W(S)^{-}=W(T)^{-}$.

(iii') $W(S)^{-w}=W(T)^{-w}$.

(iv) $W_{n}(S)^{-}=W_{n}(T)^{-}$for all $n \geqq 1$.

Note that $\left\{W_{1}(T)^{-}, W_{2}(T)^{-}, \ldots\right\}$ gives a complete set of invariants for the approximate equivalence for a compact operator $T$. We now turn to the problem of unitary equivalence of compact operators.

For any operator $T$, we define the reducing null-space of $T$ to be the intersection of the null-spaces of $T$ and $T^{*}$ and define the essential subspace for $T$ to be the subspace spanned by the ranges of all compact operators in $C^{*}(T)$. We note that for any compact operator $T$, the reducing null-space of $T$ is the orthogonal complement of the essential subspace of $T$ and that both subspaces reduce $T$.

Proof of Theorem 2. Let $T_{e}$ and $T^{\prime}$ be the restrictions of $T$ to the essential subspace and the reducing null-space of $T$ respectively. Then $T$ is unitarily equivalent to $T_{e} \oplus T^{\prime}$ and $T^{\prime}$ is a zero operator. We decompose $S$ in a similar way. Now if $W_{n}(S)^{-}=W_{n}(T)^{-}$for all $n \geqq 1$, it follows from Theorem 1 that $S$ is approximately equivalent to $T$. According to Theorem 5 of [4], this implies that $S_{e}$ is unitarily equivalent to $T_{e}$ (cf. [13], [23]). Also since the reducing null-spaces of $S$ and $T$ have the same dimension, we see that $S$ is indeed unitarily equivalent to $T$.

We remark that the collection $\left\{W_{n}(T): n \geqq 1\right\}$ together with the dimension of the reducing null-space of $T$ constitute a complete set of unitary invariants for a compact operator $T$.

COROLlaRY (Parrott). Let $S$ and $T$ be compact operators with zero reducing nullspaces. Then $S$ is unitarily equivalent to $T$ if and only if $W_{n}(S)=W_{n}(T)$ for all $n \geqq 1$.

\section{REFERENCES}

1. W. Arveson, Unitary invariants for compact operators, Bull. Amer. Math. Soc. 76 (1970), 88-91.

2. W. Arveson, Subalgebras of $C^{*}$-algebras, Acta Math. 123 (1969), 141-224.

3. W. Arveson, Subalgebras of $C^{*}$-algebras II, Acta Math. 128 (1972), 271-308.

4. W. Arveson, Notes on extensions of $C^{*}$-algebras, Duke Math. J. 44 (1977), 329-355. 
5. F. F. Bonsall and J. Duncan, Numerical Ranges II, London Math. Soc. Lecture Notes Ser. Vol. 10, (Cambridge Univ. Press, 1973).

6. J. Brenner, The problem of unitary equivalence, Acta Math. 86 (1951), 297-308.

7. A. Brown, The unitary equivalence of binormal operators, Amer. J. Math. 76 (1954), 414-434.

8. J. Bunce and N. Salinas, Completely positive maps on $\mathrm{C}^{*}$-algebras and the left matricial spectra of an operator, Duke Math. J. 43 (1976), 747-774.

9. W. F. Chuan, Containment relations of operators and representations of $\mathrm{C}^{*}$-algebras, Dissertation, Univ. of California at Santa Barbara, 1979.

10. D. Deckard, Complete sets of unitary invariants for compact and trace-class operators, Acta Sci. Math. (Szeged) 28 (1967), 9-20.

11. D. Deckard and C. Pearcy, On unitary equivalence of Hilbert-Schmidt operators, Proc. Amer. Math. Soc. 16 (1965), 671-675.

12. J. Ernest, Charting the operator terrain, Mem. Amer. Math. Soc. 171 (1976).

13. D. W. Hadwin, An operator-valued spectrum, Indiana Univ. Math. J. 26 (1977), 329-340.

14. D. W. Hadwin, Closures of unitary equivalence classes and completely positive maps, Notices Amer. Math. Soc. 23 (1976), A-587.

15. P. R. Halmos, Introduction to Hilbert space and the theory of spectral multiplicity, 2nd Ed., (Chelsea, New York, 1951).

16. F. Murnagham, On the unitary invariants of a square matrix, An. Acad. Brasil. Ci. 26 (1954), 1-7.

17. C. Pearcy, A complete set of unitary invariants for $3 \times 3$ complex matrices, Trans. Amer. Math. Soc. 104 (1962), 425-429.

18. C. Pearcy, A complete set of unitary invariants for operators generating finite $W^{*}$-algebras of type I, Pacific. J. Math. 12 (1962), 1405-1416.

19. H. Radjavi, On unitary equivalence of arbitrary matrices, Trans. Amer. Math. Soc. 104 (1962), 363-373.

20. I. E. Segal, Decompositions of operator algebras II, Mem. Amer. Math. Soc. 9 (1951).

21. W. Specht, Zur Theorie der Matrizen, II, Jber. Dentsch. Math. Verein. 50 (1940), 19-23.

22. P. K. Tam, On the unitary equivalence of certain classes of non-normal operators, $I$, Canad. J. Math. 23 (1971), 849-856.

23. D. Voiculescu, A non-commutative Weyl-von Neumann theorem, Rev. Roum. Math. Pures Appl. 21 (1976), 97-113.

Department of Mathematics

Chung-Yuan Christlan University

Chung Li, Tatwan 320

REPUBLIC OF CHINA 